\title{
Lumen
}

Selected Proceedings from the Canadian Society for Eighteenth-Century Studies

\section{Pour une lecture biographique de la guerre chez Sade}

\section{Armelle St-Martin}

Volume 30, 2011

URI : https://id.erudit.org/iderudit/1007721ar

DOI : https://doi.org/10.7202/1007721ar

Aller au sommaire du numéro

Éditeur(s)

Canadian Society for Eighteenth-Century Studies / Société canadienne d'étude du dix-huitième siècle

ISSN

1209-3696 (imprimé)

1927-8284 (numérique)

Découvrir la revue

Citer cet article

St-Martin, A. (2011). Pour une lecture biographique de la guerre chez Sade.

Lumen, 30, 153-170. https://doi.org/10.7202/1007721ar

Copyright (C Canadian Society for Eighteenth-Century Studies / Sociéte canadienne d'étude du dix-huitième siècle, 2011
Ce document est protégé par la loi sur le droit d'auteur. L'utilisation des services d'Érudit (y compris la reproduction) est assujettie à sa politique d'utilisation que vous pouvez consulter en ligne.

https://apropos.erudit.org/fr/usagers/politique-dutilisation/ 


\section{Pour une lecture biographique de la guerre chez Sade}

Si une partie de la critique s'est évertuée à discréditer la place de la biographie dans la compréhension des œuvres littéraires, il n'en demeure pas moins que dans certains cas, il existe des croisements entre l'homme et l'œuvre qu'on ne saurait négliger. Le marquis de Sade est un exemple typique de ces cas où la vie d'un écrivain a une influence déterminante sur sa vision du monde transposée dans l'univers philosophique et romanesque. Précisons d'emblée que le cas de Sade est à cet égard exceptionnel. Ce ne sont pas uniquement, à la manière d'un Molière ou d'une George Sand, des épisodes concrets qui, de l'expérience réelle, sont reportés dans l'univers de la fiction. Mais c'est véritablement l'ensemble des vies de la lignée des Sade, nous voulons parler de cet héritage ancestral - devenu objet concret par la figure du père et les écrits qu'il a légués à son fils - qui façonne le rapport de Sade au monde, lequel va s'épanouir dans l'invention d'un univers romanesque et dans l'élaboration d'une pensée philosophique et morale.

Je tâcherai d'éclairer ici l'idée de la guerre chez Sade par la biographie $^{1}$. Il ne s'agira pas de revisiter des moments vécus de l'histoire personnelle du marquis (par exemple sa carrière militaire), mais plutôt de montrer la prégnance du conflit dans son roman et son théâtre par une analyse du legs paternel qui, dépassant la personne du père, le comte de Sade, remonte à l'âge d'or de la féodalité, moment où naît le nom de Sade, et qui se prolonge par sa lecture de la philosophie de Hobbes. Je ne propose pas non plus de mener une approche psychanalytique, ancrée dans la longue durée, bien que ma description semble

1 Je renvoie aux trois plus récentes biographies qui font autorité : Maurice Lever, Donatien Alphonse François, marquis de Sade, Paris, Fayard, 1991 ; Laurence L. Bongie, Sade: A Biographical Essay, Chicago \& London, The University of Chicago Press, 1998; Neil Schaeffer, The Marquis de Sade, New York, Alfred A. Knopf, 1999. 
prendre quelquefois l'allure de la reconstitution d'un mythe personnel. C'est essentiellement dans le cadre d'un texte de Jacques Derrida que s'ancre mon approche. L'impulsion théorique fondamentale voulant qu'un texte n'est tissé que de différences et qu'il n'y a pas de hors texte, permet de réévaluer, sur des bases originales, le lien toujours fructueux, mais généralement déconsidéré depuis la «Nouvelle Critique», entre l'œuvre et le «bio-graphique». La réflexion de Jacques Derrida s'est développée dans ce sens à propos de Nietzsche qui «fut le seul [...] à traiter de la philosophie avec son nom, en son nom ${ }^{2} \gg$. À partir de cette posture critique, plus riche et plus souple, qu'un aller-retour perpétuel entre ce qu'a pu vivre l'auteur et ce qu'il a écrit, il sera possible de donner un nouveau sens à la guerre, un sens qui la soustrait à l'imaginaire pornographique (on la réduit trop souvent à une métaphore de la rencontre amoureuse) pour la rattacher aussi au destin du patronyme de Sade.

Interpréter la guerre dans l'univers sadien à la lumière de la notion derridienne de «signature» nécessite ainsi de faire entrer dans la dynamique biographique autant l'expérience du marquis de Sade que celle des siens. Contrairement à une lecture traditionnelle, la seconde est aussi signifiante que la première, pour ne pas dire plus. Ce qui veut dire que la vie du père se superpose - non pas nécessairement dans un sens psychanalytique, puisque la relation qui nous intéresse s'incarne essentiellement dans la matérialité de textes écrits - à celle du fils, les œuvres de l'un et de l'autre formant un tissu unique. À propos de cette présence d'un texte dans un autre, une approche plus orthodoxe se serait donné beau jeu de dissocier l'origine de l'aboutissement, comme fin ultime de l'effort critique. Or je n'analyse les écrits du père séparément de ceux du fils que dans le but de montrer la surimpression des uns sur les autres ; ma lecture en deux temps distincts, incontournable en raison du caractère linéaire de l'écriture, n'étant jamais qu'un moyen.

La révolte du marquis - qui fera de l'intégralité de son existence une guerre qu'il a crue juste parce que, à la manière de ses ancêtres chevaliers, elle l'oppose seul à des pouvoirs qui prétendent le réduire, lui, un individu fier, indépendant et libre, à l'obéissance à l'institution monarchique - est à la fois pathétique et brave. Un tel combat est aussi celui que Sade choisit de faire vivre aux héros d'Aline et Valcour, de Jeanne Laisné, d'Adélä̈de de Brunswick et d'Isabelle de Bavière, dans

2 Jacques Derrida, «Otobiographie de Nietzsche», dans Claude Lévesque et Christie V. McDonald (éd.), L'oreille de l'autre. Otobiographies, transferts, traduction. Textes et débats avec Jacques Derrida, Montréal, Vlb éditeur, 1982, p. 18. 
une moindre mesure. Que ces textes soient ceux avoués par Sade et que trois d'entre eux soient des œuvres historiques, campées dans le Moyen Âge et mettant en scène des conflits armés, n'est pas insignifiant. Si Sade choisit de faire porter sa signature à ces œuvres, c'est peut-être parce qu'elles constituent la meilleure élaboration de sa biographie, de ses origines au XII ${ }^{\mathrm{e}}$ siècle jusqu'au présent de son drame dans les prisons. Écrits par lui et pour parler de lui (ce qui inclut ses ancêtres), en montrant où se situe son identité propre, mais en passant par le détour de l'invention romanesque, très souvent à la troisième personne, les quatre textes que je viens de mentionner se lisent ainsi à la fois comme une biographie et une autobiographie, comme avatars donc de $1^{\prime}$ «otobiographie ${ }^{3} »$ derridienne. Une telle représentation de Sade dans la fiction, tout en étant indissociable du glorieux patrimoine ancestral, se double de zones qui paraissent sombres et inquiétantes à un lecteur qui ne serait pas averti. Ces zones demeurent en retrait dans les œuvres signées, ce qui ne diminue en rien leur importance - bien au contraire - et elles s'avancent à visage découvert dans les romans anonymes comme Justine et Juliette. Même si elles apparaissent avec un relief moins prononcé dans Aline et Valcour et les textes historiques, nous ne pouvons les ignorer. $\mathrm{D}^{\prime}$ une manière parallèle, mais pas équivalente à ce que Jacques Derrida a pu montrer pour Nietzsche dont le père porte un poids, une responsabilité dans sa vie, ces zones que je viens de qualifier de ténébreuses se rattachent au nom du père et à cet héritage où le seigneur, par sa force, pouvait imposer, en toute impunité, l'arbitraire de son pouvoir au plus faible. Aucun projet de rédemption n'est évidemment envisagé par Sade et il n'a pas tenté de camoufler l'injustice sociale, devenue le plus grand crime au moment où il écrit après la Révolution, par une philosophie matérialiste de la nature, de la nature humaine et du conflit. Celle-ci sert au contraire à établir la vérité et le bien-fondé de la domination féroce. Le nom de Hobbes, plus que tout autre, permet à Sade de jeter un regard lucide sur son instinct guerrier et celui de ses ancêtres dans un effort non pas uniquement de justification, mais aussi et surtout de revalorisation de la violence. On ne peut douter qu'il y ait là autant de provocation que de mauvaise foi. Sade sait parfaitement que la brutalité de certains de ses comportements n'engage que lui, il reconnaît et chérit le caractère foncièrement

3 Jacques Derrida insiste sur une «totale redistribution» du problème de l'autos, de l'autobiographique, qui «oblige non pas à dissoudre la valeur de récit autobiographique, mais à la re-structurer autrement à partir d'un projet qui est aussi biographique ou thanatographique» (Jacques Derrida, «Otobiographie de Nietzsche», op. cit., p. 64). 
exceptionnel de sa personnalité, mais en même temps il s'obstine à faire passer ses actions pour la norme. Si on garde à l'esprit le poids du passé féodal et militaire de Sade, on peut imaginer qu'il aurait été difficile qu'il pût concevoir les relations humaines autrement que comme une guerre perpétuelle où il devient juste que le faible succombe pour le plaisir ou l'utilité du plus fort.

Dans la conférence «signature événement contexte», Jacques Derrida se livre à une réflexion sur l'écriture comme moyen de communication. Ce travail s'inscrit dans la foulée de ses idées antérieures sur l'opposition parole / écriture dans la tradition philosophique. Dans ce texte, il s'intéresse tout particulièrement à la marque que constitue la signature. Derrida souligne ceci : «une signature doit avoir une forme répétable, itérable, imitable ; elle doit pouvoir se détacher de l'intention présente et singulière de sa production ${ }^{4}$.» En effet, la signature écrite implique à la fois la «non-présence actuelle ou empirique du signataire ${ }^{5}$ » et marque un «avoir-été présent dans un maintenant passé, qui restera un maintenant futur, donc dans un maintenant en général, dans la [...] forme transcendantale de la maintenance ${ }^{6} . »$ Cette présence-absence qui traverse la signature, pourfend aussi l'écriture qui - ici Jacques Derrida s'appuie sur les marges de l'Essai sur l'origine des langues de Condillac — doit «pouvoir être répétée ${ }^{7}$ ». Du coup, l'écriture, étant itérable, peut porter une signification différente de celle où elle a été émise ; elle doit ainsi signifier dans plus d'un contexte. On voit dès lors les implications sur le sujet énonciateur, sur l'auteur qui signe son texte. Ce dernier échappe invariablement à son signataire qui ne peut prétendre maintenir la signification de ce qu'il a signé. À la fois dans le texte et hors du texte, la signature loin uniquement de sceller un sens voulu par l'auteur, ouvre aussi la voie à la dissémination du sens.

Plus tard, à propos de Nietzsche, Jacques Derrida reviendra sur ce concept de signature pour l'associer à l'autobiographie. Dans «Otobiographies de Nietzsche», il pourra ainsi ouvrir un nouvel horizon pour la biographie à travers cette réévaluation critique de la notion de «signature». Sous cet angle, la biographie peut en effet prendre ses distances face aux données empiriques (celles fournies par la psy-

4 Jacques Derrida, Marges de la philosophie, Paris, Les éditions de Minuit, 1972, p. 392.

5 Ibid., p. 391.

6 Ibid., p. 391.

7 Ibid. p. 371. 
chologie ou la psychanalyse, l'histoire ou la société) pour «mobiliser une analyse du nom propre et de la signature ${ }^{8} »$. Du coup, l'œuvre et la vie sont unies, mais non pas en se confondant entièrement l'une avec l'autre, mais en partageant plutôt une bordure qui permet le passage de l'une vers l'autre, qui fait que l'une se construit grâce à l'autre, et qui marque dialectiquement l'émergence du moi. Jacques Derrida pourra ainsi à partir de l'autobiographie Ecce Homo envisager la lecture d'un texte nietzschéen à caractère politique. Dans ce cas précis, la lecture de Nietzsche s'enrichit par le recours «à ce que Nietzsche enseigne luimême du "crédit" ouvert à une signature, [...], de la différance posthume entre lui et son œuvre, etc. ${ }^{9} \gg$

Cette démarche pose bien entendu des problèmes. L'objection principale que l'on pourrait porter renvoie à l'autonomie et à la cohérence propre d'un écrit politique, fortement marqué par son genre. Le détour par l'autobiographie ne viendrait-il pas jeter un voile sur les conceptions théoriques de l'auteur? Amène-t-il véritablement à une meilleure compréhension du texte ciblé? Toutefois, et en m'appuyant sur la remarque de Béatrice Didier - «l'écriture autobiographique est [...] l'écriture par excellence ${ }^{10}{ }_{\gg}-$ dans ces cas où un auteur entreprend d'écrire sa biographie, je dirais que cet acte de mettre sa vie en scène doit être intégré à notre effort pour interpréter l'œuvre.

Par ailleurs, et pour en revenir à Jacques Derrida lisant Nietzsche comme point d'ancrage de mon article, le lien entre Sade et Nietzsche ne va pas nécessairement de soi, bien qu'il existe des affinités idéologiques certaines entre les deux hommes. La question de la mère est un exemple qui témoigne de la distance qui les sépare. Jacques Derrida a intimement mêlé la question du père à celle de la mère. En fin de compte, le père est associé, dans la pensée de Nietzsche, à la dégénérescence historique liée à la langue maternelle. Pour Sade, le problème de la mère se pose tout autrement. Contrairement à Nietzsche, il a explicitement et consciemment rejeté la figure maternelle, pour mieux s'offrir le plaisir, toujours renouvelé, de l'éventrer. La symbolique liée au meurtre de la mère dans la fiction divise les critiques. Ce qui est sûr, c'est que dans ses lettres, il n'a cessé de vitupérer contre elle. La force de cette haine est équivalente à celle de son attachement pour son père. Le rejet de la mère est un acte que Sade a constamment signé, comme il a lucidement

8 Jacques Derrida, «Otobiographie de Nietzsche», op. cit., p. 16.

9 Ibid., p. 37.

10 Béatrice Didier, Stendhal autobiographe, Paris, Presses universitaires de France 1983, p. 46. 
endossé la signature paternelle. Le ton vertueux, sage et pondéré du comte dans ses écrits n'a en rien éloigné le fils qui, comme le souligne Maurice Lever, «ne cessera, tout au long de sa vie de [...] lire et de [...] relire ${ }^{11} \gg$ les manuscrits de son père. Alors que chez Nietzsche le père renvoie ultimement à la figure d'un État abhorré ${ }^{12}$, chez Sade il a la valeur fondatrice des utopies politiques. Toutefois, et malgré cette différence, il m'a paru que, comme Nietzsche, la signature (qu'elle soit finalement «vomie» ou intériorisée favorablement) était une donnée fondamentale. Et le choix de Sade de signer ou de ne pas signer ses œuvres avait une signification à laquelle seule l'approche derridienne donne une portée qui ne soit pas simplement contingente. Il devient alors possible d'expliquer l'œuvre sadienne au prisme de la patrilinéarité de son auteur, en dépit du fait que les romans pornographiques, moins avouables, plus controversés, ne soient pas signés, alors que les textes plus «lisibles» le sont. Un tel argument est sans doute vrai. Par contre, si on s'y arrête, il devient difficile d'attacher un sens original à l'influence réelle exercée par le père et le nom du père. Influence démontrée par tous les biographes de Sade, mais dont on a sous-évalué la portée sur les textes sadiens qui se situent en dehors du genre licencieux. En conjuguant l'idée de la signature, à la fois comme même et différente (que ce soit le fils ou le père qui signe, il s'agit toujours de la signature des Sade), et la notion d'autobiographie comme espace de rencontre entre le vécu et le texte signé (la signature apposée au bas des écrits du fils est le trait qui unit et confond les expériences passées du père à l'actualité de celles du marquis), on peut proposer une lecture de Sade qui dépasse le contexte restreint, premier et évident des œuvres. La marque laissée sur le papier qui rattache Sade au père mort, dans les écrits du comte, devient alors signifiante dans la mesure où elle permet d'identifier ces écrits comme un «protocole», c'est-à-dire de leur assigner la même fonction que Jacques Derrida a assigné à Ecce Homo, une clé qui permet de mettre au jour la signification de l'œuvre politique nietzschéenne. Je verrai ainsi dans les écrits du père la clé offrant un accès à l'univers du fils.

Voyons rapidement le tableau de cet héritage, ces «Écrits paternels» pour leur donner un titre, laissé par le comte de Sade à son fils pour son édification morale. L'œuvre du comte contient de longs passages qui

11 Maurice Lever, «Introduction» in Marquis de Sade, Écrits politiques, Paris, Bartillat, 2009 , p. 30.

12 Jacques Derrida, «Otobiographie de Nietzsche», op. cit., p. 51. 
constituent ses réflexions sur la vie politique de son époque. Les commentaires sur la guerre sont nombreux et suivis. Ils ont la cohérence d'une pensée idéologique. Et c'est moins en stratège que le comte parle de la guerre qu'en moralisateur. Aussi jette-t-il un regard critique sur les conflits armés. Il connaît, pour les avoir vécus, les ravages qu'ils causent lorsqu'ils sont entrepris uniquement pour satisfaire les ambitions de quelque ministre, peu soucieux du bien-être de État et du peuple. Il reprend ainsi certaines idées de Voltaire, avec qui il correspondait. Il n'est pas loin d'affirmer, malgré une prudence toute rhétorique, que les guerres sont le témoignage de l'irrationalité humaine en matière de politique : "Que de sang répandu dans cette dernière guerre pour la maison d'Autriche, après en avoir tant répandu contre elle! On eut raison alors, on a raison aujourd'hui. Si je regrette ce qu'il en a coûté $\mathrm{d}^{\prime}$ hommes et d'argent, ce n'est pas pour critiquer ce qu' on a fait ${ }^{13}$.»

Et s'il doit y avoir une guerre, celle-ci doit être menée de sorte à épargner les pertes de vie inutiles. D'où l'importance de la stratégie militaire, au détriment des actes, qualifiés de bravoure, mais qui ne visent qu'à assurer la gloire du seul individu qui en a pris l'initiative. Le comte de Sade s'appuie sur l'exemple de la perte du Canada au cours de la guerre de Sept Ans :

Il ne suffit pas d'être brave quand on commande. M. de $\mathrm{V}^{* * *}$ [Vaudreuil] pouvait envoyer le secours, mais il voulut le conduire pour partager la gloire avec M. de $\mathrm{M}^{* * *}$ [Montcalm]. La vanité et l'ambition de ces deux hommes ont perdu nos affaires dans ce pays dont l'importance est constatée par les efforts qu'ont fait les Anglais pour nous le ravir, et dans ceux qu'ils feront pour le garder ${ }^{14}$.

À lire les écrits militaires du père de Sade, on sent que sa condamnation de la guerre est intimement liée à une forte réprobation de la vanité et de l'ambition. Ces dernières causent un étiolement des vertus guerrières : «Les principes militaires sont perdus, se lamente-t-il. Il n'y a plus ni discipline, ni émulation, ni honneur, ni concorde, ni subordination, ni bonne foi, ni grandeur d'âme, ni noblesse, ni sentiment : tout est pour soi ${ }^{15}$.» Le comte souhaite voir dans les rangs de la noblesse française plus de fierté et une plus grande capacité de se dépasser dans

13 Marquis de Sade, Écrits politiques, op. cit., p. 31.

14 Ibid., p. 68.

15 Ibid., p. 68. 
un idéal au service de la patrie. Il se lamente aussi que la guerre, en son siècle, soit devenue un instrument servant à manipuler un prince. Et ni Louis XIV, ni Louis XV n'échappent à ses jugements défavorables. À l'un il reproche son despotisme, à l'autre sa mollesse.

À lire les romans pornographiques du marquis de Sade, il apparaît $\mathrm{d}$ 'abord que la morale du père a eu peu d'effet. On pourrait même dire que La Nouvelle Justine et l'Histoire de Juliette offrent l'exact démenti des thèses paternelles ; les libertins de ces deux romans font de la tyrannie, de l'égoïsme et de la lâcheté les mots d'ordre de leur existence. Est-ce à dire que la valeur de "protocole» que j'attribue aux écrits du comte doit être remise en question? Si on se replace dans la logique derridienne de la signature, il faut se tourner vers les œuvres signées pour pouvoir poser un jugement nuancé. Ce sont plutôt ces textes, et moins les romans libertins anonymes, qui portent l'identité de Sade. Or, Aline et Valcour et les œuvres historiques sont, sans surprise, une illustration fictionnelle des idées du père sur la guerre. À l'instar des cogitations idéalistes paternelles, les héros de Jeanne Laisné et d'Adélaïde de Brunswick sont des guerriers fiers pour qui le renoncement à soi pour servir la patrie est un devoir et une question d'honneur. Lors du siège de Beauvais, Jeanne, qui bientôt prendra la tête de la défense de la ville, aidée de toutes les femmes, peint ce portrait d'elle-même au traître Chimai, au nom illustre, mais vendu au duc de Bourgogne :

\footnotetext{
Ah! connais mieux mon cœur, il n'est pas fait pour toi ;

Il n'est pas fait pour vendre et pour trahir son roi.

Ta foi ni ton amour, ton nom ni tes richesses

Ne me feraient jamais partager tes bassesses.

Je suis femme et française... apprends-le, séducteur,

Ma patrie est mon bien, mon trésor est l'honneur ${ }^{16}$.
}

Bien que dans ses écrits le comte se pose comme un adversaire de la monarchie absolue, il n'en demeure pas moins qu'il reste attaché au service de son roi. Mais il chérit par-dessus tout sa patrie, la France, qu'il affirme «capable de grandes choses sous un grand prince ${ }^{17}$ ».

La même fierté et le même courage dans la guerre caractérisent Adélaïde. Lorsque l'empereur menace la souveraineté de la Saxe, la reine,

16 Sade, Jeanne Laisné, in Théâtre 1, Pauvert, Paris, 1991, p. 134.

17 Marquis de Sade, Écrits politiques, op. cit., p. 59. 
malgré son jeune âge, fait preuve d'une énergie peu commune dans ses paroles :

[E]lle disait à l'empereur que ce n'était ni à son âge, ni avec la dépravation de sa conduite, qu'on devait se flatter d'imposer au prince toujours victorieux qui régnait sur la Saxe et qu'avant de se permettre une telle audace, il fallait que Henri réformât premièrement ses mœurs, en chassant ses maîtresses, et son insolence en sortant aussitôt de l'état d'un prince qui n'avait nul besoin de rétablir l'ordre chez lui, qu'en sa qualité d'empereur il ferait beaucoup mieux d'aller faire régner dans ses autres provinces, lesquelles, bien plus que la Saxe, avaient besoin de ses conseils et de ses secours ${ }^{18}$.

Dans Adélä̈de de Brunswick ou dans Jeanne Laisné, le monarque renvoie à une figure pâle, sinon absente. Les personnages qui brillent dans ces textes sont les héros solitaires du domaine seigneurial.

Cet esprit de grandeur qui anime la noblesse est présent de manière oblique dans Isabelle de Bavière. Si les rênes du royaume de France sont revenues à la perfide Isabelle, et si le pays a sombré dans la guerre civile, le manque de courage du roi est à blâmer. Toutefois, de nombreux chevaliers n'hésitent pas à se sacrifier pour protéger la France des manigances néfastes de la reine avec les Anglais, malgré le fait que le climat général qui règne à la cour est celui d'une lutte des ducs pour le pouvoir.

Il est significatif que Sade ait choisi de camper la fierté nobiliaire dans des œuvres historiques relatant un conflit armé. Comme l'a jugé son père, le temps présent est dégénéré et ce n'est que le passé qui offre des exemples de vertus. En outre, c'est sur le champ de bataille que la noblesse prouve sa valeur et non pas à la cour. À cet égard, Adélaïde de Brunswick est exemplaire par l'éclairage défavorable que le marquis jette sur Mersbourg, figure du courtisan, qui ne songe qu'à ses propres intérêts en intriguant à la fois auprès du roi, de la reine et de leur homme de confiance, le marquis de Thuringe, quitte à mettre en péril la sécurité du royaume.

Très tôt dans le développement des études sadiennes, Béatrice Didier a montré l'importance du Moyen Âge dans l'œuvre de Sade ${ }^{19}$. Cette époque, réputée barbare, lui offre tout naturellement des motifs

18 Sade, Adelaïde de Brunswick, in CEuvres complètes, Cercle du livre précieux, Paris, tome 13, p. 140.

19 Béatrice Didier, «L'image du Moyen Âge chez Sade», L'image du Moyen Age dans 
pour nourrir son roman noir, à commencer par le château, indispensable à la «féodalité du plaisir ${ }^{20}{ }^{2}$. La prédilection de Sade pour les héros du Moyen Âge s'explique aussi par son histoire ancestrale. C'est bien à cette époque que la famille de Sade commence à vivre un âge d'or. Et cette mémoire est si bien cultivée qu'il est aisé de reconstituer avec précision, à partir du $\mathrm{XI}^{\mathrm{e}}$ siècle, le moindre fait héroïque de la lignée. Le marquis qui a grandi au milieu des parchemins et des archives familiales est bien conscient du prestige associé à son nom et il revit, à travers ses héros et ses héroïnes, ce passé glorieux où les seigneurs Sade, riches et puissants, régnaient sur les fiefs de Saumane et de Mazan.

Il serait difficile de réduire à la nostalgie le sentiment qui anime Sade lorsqu'il compose ses œuvres historiques. Il faut voir dans ces mises en scènes historiques, un dégoût profond pour l'époque actuelle, qui oppose la loi à la volonté capricieuse de l'aristocratie. Sade, marqué depuis son enfance par les prérogatives liées à sa caste, a fortement intériorisé, à cause de sa personnalité naturellement despotique et dominatrice, le pouvoir sans limite du seigneur, transmis sans interruption au fils aîné de la famille depuis plus de 500 ans. Du coup, à l'image de quelques-uns de ses ancêtres chevaliers qui devaient se défendre seuls, Sade croit mener une guerre héroïque et solitaire, non plus contre un ennemi réel, mais contre la superstition et les préjugés tels qu'ils $\mathrm{s}^{\prime}$ incarnent dans la religion, les mœurs, la morale et la loi. François Ost a bien souligné la lutte continuelle et acharnée de Sade contre le système judiciaire ${ }^{21}$. Peut-être en a-t-il négligé le pathétique, imputable à l'isolement du marquis et à son inébranlable conviction du bien-fondé de sa lutte. Ce qu'il tente désespérément de faire entendre, c'est que ses désirs sont non seulement dans la nature, mais que c'est aussi son droit, de par sa position sociale, de les satisfaire. C'est avec cette conscience aiguë de la supériorité de sa caste que Sade a mené son «combat», lutte à laquelle il a tenté de donner une couleur universelle par une réflexion sur le gouvernement et la loi.

Pierre Favre a bien tenté une synthèse des idées politiques de Sade par une lecture des utopies de Butua et de Tamoé, insérées au cœur d'Aline et Valcour. Au terme de son essai, Pierre Favre accorde aux idées politiques de Sade la valeur d'une «curiosité». Valeur qui trouve son fondement dans le fait que Sade «est un personnage hors du commun,

la littérature française de la Renaissance au XX $X^{e}$ siècle, Poitiers, Faculté des lettres de Poitiers, 1982, p. 301-311.

20 Béatrice Didier, Sade. Une écriture du désir, Paris, Denoël /Gonthier, 1976, p. 23.

21 François Ost, Sade et la loi, Paris, Odile Jacob, 2005. 
tant psychologiquement que moralement ${ }^{22} \gg$. Ceci ne signifie pas $\mathrm{du}$ tout que la pensée de Sade est stérile. Pierre Favre l'admet. Cette fertilité vient, à mon sens, de la facette enchantée, pour ainsi dire, de cette politique qui, développée dans l'univers fictionnel à la manière d'un rêve, joue sur la disparition fantasmatique de l'État.

L'opposition entre le héros solitaire, inébranlable dans ses convictions, et la multitude, basse et rampante, est un motif constant des œuvres signées alors qu'un roman comme l'Histoire de Juliette s'organise autour des associations de criminels. Aline et Valcour constitue le meilleur exemple de la lutte de l'individu contre la société, exemple d'autant plus convaincant que la forme épistolaire laisserait présager un éclatement de l'unité romanesque. Or, malgré la multiplication des points de vue ou des histoires, Sainville (et son double Léonore) demeure le protagoniste vers lequel l'attention du lecteur est constamment tournée. Homme d'action, il mène seul une lutte contre le destin et les hommes pour retrouver la femme qu'il aime. En ce sens, Adélä̈de de Brunswick ne fait que dédoubler Aline et Valcour : Frédéric de Saxe, accompagné d'un seul chevalier, parcourt inlassablement ses États et ceux voisins à la recherche d'Adélaïde. Bien qu'il soit légitime de ne voir dans la quête $\mathrm{du}$ prince qu'un pastiche des romans de chevalerie, il n'en demeure pas moins que la solitude profonde de ce héros, ses manifestations de cruauté, son esprit vindicatif servent à le rapprocher de Sade. D'où le côté inquiétant de ces héros révoltés. Car tous, à l'exception de Jeanne Laisné, poursuivent des buts égoïstes. Leur lutte est intimement associée à leurs propres désirs. Que ceux-ci prennent la forme du sacrifice du bien de l'État, comme c'est le cas pour la reine Isabelle de Bavière et pour le prince de Saxe, ou que ces fins s'incarnent dans le renoncement à tout code moral, Sainville dans Aline et Valcour en est le témoignage, $c^{\prime}$ est toujours dans le besoin d'assurer leur propre bonheur, peu importe les conséquences, que ces personnages puisent l'énergie du combat. De ce point de vue, leurs actes héroïques doivent aussi se comprendre comme des manifestations de leur individualisme. En fin de compte, leur statut de noble guerrier passe au second plan pour expliquer leur bravoure. Celle-ci dérive de leur nature intéressée.

J'avais mentionné plus haut les réflexions du comte de Sade sur ce sujet dans ses écrits sur la guerre. Il avait mis au compte des ambitions personnelles la déchéance militaire de la France à la suite de la guerre de Sept Ans. Sade s'intéressera à cette question d'un point de vue phi-

22 Pierre Favre, Sade utopiste. Sexualité, pouvoir et État dans le roman "Aline et Valcour», Paris, Presses universitaires de France, 1967, p. 100. 
losophique et s'appuiera sur Hobbes. J'y reviendrai. La gloire de l'État, le dévouement à sa patrie n'ont aucune place dans cette vision du monde. Il semble que, contrairement aux idées de son père, l'absence de ces idéaux ne constitue en rien une dépravation ${ }^{23}$. Le comte de Sade rejetterait donc ce matérialisme, que Sade a exemplifié dans Aline et Valcour? On ne peut répondre affirmativement à cette réponse. Il faut se souvenir qu'au cours de sa jeunesse le comte s'est jeté dans la lutte pour le pouvoir à la cour de Louis XV. Peu scrupuleux, seuls ses intérêts lui importaient et il ne s'est jamais embarrassé d'une morale. Le marquis de Sade était parfaitement au courant de la jeunesse trouble de son père. Aline et Valcour, qu'on a toujours dit autobiographique (mais dans un sens traditionnel) ne porte pas vainement la signature de Sade. Et ce n'est pas uniquement le «je» de sa personne individuelle qui se prononce pour cette philosophie ; il la revendique pour tout son clan, par sa signature. Celle-ci permet ainsi de jeter un regard neuf sur ce texte et autorise à chercher un sens qui dépasse une lecture des faits empiriques de la vie de l'auteur rapportés, peu importe leur degré de modification, dans l'univers fictionnel.

Mais ces écrits du père ont certainement, eux aussi, une valeur empirique? Il est bien vrai qu'ils ont une réalité semblable à celle du passage de Sade dans l'armée, alors qu'il avait quatorze ans. Toutefois, ces écrits forment un tissu textuel dont la permanence est bien plus réelle qu'un épisode singulier de la vie, toujours soumis au passage du temps qui n'en traduit qu'une signification qui demeure instable pour la conscience. Par contre, on ne peut se contenter d'un va-et-vient linéaire entre la pensée du comte et celle du marquis ; une image fragmentaire de l'œuvre serait la conséquence de ce manque de souplesse dans l'analyse. En donnant une signification à la signature et en problématisant l'identité des textes du père et du fils, on saisit mieux le caractère fondamental du conflit chez Sade.

La domination violente lie ainsi Sade au passé de ses ancêtres féodaux. Béatrice Didier en était aussi arrivée à cette conclusion dans sa lecture des motifs du château dans les œuvres licencieuses ${ }^{24}$. Aline et Valcour, de nature autobiographique et roman épistolaire tout à fait

23 L'article que Christophe Cave consacre à Aline et Valcour va dans ce sens («Barbarie» in Sade en toutes lettres, Ed. Michel Delon et Catriona Seth, Paris, Desjonquères, 2004, p. 141-153). Le drame d'Aline s'explique par un attachement à un idéal aveugle, alors qu'une Léonore sait bien «voir» le mal logé dans le cœur de l'homme. 
avouable, développe aussi largement ce thème. C'est dans ce texte que les remarques de Sade sur la guerre sont les mieux suivies et c'est là que le lecteur est invité à pénétrer dans l'univers politique de la brutalité institutionnalisée de l'État. Jusqu'à présent, le rêve d'un paradis perdu, reconstitué par l'écriture et le retour à des origines illustres donnait corps à la violence d'Isabelle de Bavière ou d'Adélä̈de de Brunswick. Les réflexions surannées et nostalgiques du père de Sade semblent ainsi mieux convenir à l'univers révolu des œuvres historiques qu'à la tourmente des années 1780 dans laquelle est campée Aline et Valcour. Dans ses œuvres fictionnelles, Sade a toujours mêlé sa voix à celles des philosophes pour mieux exhiber, en quelque sorte, ses croyances scandaleuses, la signature d'autrui jouant le rôle de faire-valoir. Un des passages d'Aline et Valcour les plus difficiles à lire est celui du royaume des cannibales de Butua. Non seulement parce que la violence qui y est décrite choque la sensibilité, mais aussi parce que la mise en scène des signatures y est complexe. La description de Butua, qui ne fait pourtant que quelques dizaines de pages, est criblée de notes de bas de page qui renvoient à Montesquieu, Raynal, Cook, Démeunier, Lucrèce, Helvétius, etc. ${ }^{25}$, avec lesquels Sade, en tant qu'auteur, entre dans un véritable dialogue. En premier lieu pour établir clairement son appartenance aux savants et au clan des esprits éclairés, qui dénoncent les préjugés et remettent en question la morale au nom de la relativité des mœurs, ensuite pour se mettre en scène, lui et son nom (le «nous» est omniprésent), de sorte que le lecteur ne puisse pas oublier que le «je» de Sade, virtuel pour ainsi dire, existe bien derrière le «je» réel de la voix de Sainville, porteur du récit et figure héroïque «de l'antique chevalerie ${ }^{26}$ ». Il existe un «absent» notoire dans cette description : Hobbes $^{27}$. Sade ne le nomme jamais dans le portrait des cannibales et pourtant son matérialisme est au fondement de l'organisation étatique de Butua. En général, la critique sadienne s'est peu penchée sur la présence de Hobbes dans le texte sadien. À l'exception des renvois

25 Pour une analyse précise des emprunts de Sade, voir : Michel Delon, «Notes et variantes» in D.-A.-F. Sade, CEuvres, Paris, Gallimard, Bibliothèque de la Pléiade, 1990, tome 1, p. 1229-1354.

26 Sade, Aline et Valcour ou le roman philosophique, CEuvres, Ed. Michel Delon, Paris, Gallimard, 1990, tome 1, p. 423.

27 Le marquis possède à La Coste un exemplaire du Citoyen. Il a aussi pu lire le long article que Diderot consacre à Hobbes dans l'Encyclopédie. Par ailleurs le Système de la nature de d'Holbach, que Sade connaît fort bien, contient des résumés de la pensée de Hobbes. 
que Michel Delon a signalés dans ses «Notes» d'Aline et Valcour, on s'est peu soucié d'approfondir cette filiation ; la raison en est peut-être que Sade n'a pas plagié des passages de Hobbes, comme il a pu le faire pour d'autres auteurs. Comment comprendre cette absence du nom propre de Hobbes, alors qu'Aline et Valcour est l'illustration parfaite de l'homme naturel du Léviathan? En tenant compte du fantasme de Sade d'un régime politique de la violence et en gardant en tête l'étendue des développements qu'il consacre au meurtre, on peut dire que Hobbes constitue bien la conscience politique du marquis de Sade, son alter ego philosophique, un autre «je» en quelque sorte. Et semblablement à toute autobiographie où l'écrivain qui écrit sa propre vie ne nomme son nom propre qu'au moment où il signe son texte $-\mathrm{le}$ «je» du récit ne peut désigner que l'auteur lui-même - semblablement donc, Sade n'a pas nommé celui qui incarne sa voix au cœur de son roman. Par conséquent, le lecteur qui tâche de reconstituer la pensée de Sade doit être prudent. Les philosophes qu'il nomme (un d'Holbach, un Machiavel ou un La Mettrie) ne sont pas nécessairement ses meilleurs porteparoles. Béatrice Didier a bien souligné, dans le cas de l'autobiographie chez Stendhal, que le «je» réunissait deux «moi» différents au niveau de la temporalité : le moi de «l'écrivain en train d'écrire et l'homme qui a $v \mathrm{cu}^{28}{ }^{2}$. Dans une logique derridienne, cette différence ne pourrait-elle pas aussi porter sur l'identité radicale du «je» qui serait double dans l'immédiateté de l'écriture, le «je» du locuteur Sainville désignant à la fois Sade et Hobbes? On comprend dès lors mieux la mise en scène de la pulsion guerrière de l'homme. Aspect essentiel de la philosophie de Hobbes, elle est pleinement assumée par Sade qui choisit «le mode de la fiction rationnelle» ${ }^{29}$, à l'instar du philosophe anglais, pour théâtraliser la nature humaine.

Béatrice Didier, Stendhal autobiographe, op. cit.

29 C'est le terme qu'emploie François Dugré pour décrire l'effort de Hobbes brossant un portrait de l'homme naturel dans le Léviathan («Fictions anciennes et modernes du politiques», Tangence, no. 63, 2000, p. 35). Le recours à la fiction serait donc un autre trait rapprochant Sade et Hobbes (la fiction de Rousseau sur l'homme naturel n'a causé que de la répugnance chez Sade). Kinch Hoekstra aura aussi été sensible au fait que Hobbes recourt à l'imagination, voire à une certaine rhétorique, pour accentuer la misère de l'homme naturel dans le but d'exhorter ses lecteurs à respecter l'ordre établi (Kinch Hoekstra, «Hobbes on the Natural Condition of Mankind», dans Patricia Springborg (éd.), The Cambridge Companion to Hobbes's «Leviathan», New York, Cambridge University Press, 2007, p. 109-127. 
La violence n'est donc pas propre à l'enfer des cannibales africains, bien que ce soit dans ce royaume qu'elle est la plus criante ${ }^{30}$. Ainsi que ce soit dans le paradis utopique de l'île de Tamoé, ou dans le raffinement des salons français, un instinct, qu'aucune loi ne freine, détermine l'homme vers la domination violente de son semblable. Cette idée fondamentale de l'universalité de cet instinct destructeur, cette «barbarie», survivant dans le cœur de l'homme en société a séduit Sade et le principe hobbesien, à savoir que l'homme est un loup pour l'homme, parcourt Aline et Valcour d'un bout à l'autre.

La guerre constitue l'unique topos de la rencontre de Sainville avec les sauvages de Butua. D'emblée, c'est un détail militaire, les fortifications, qui frappe le jeune Européen :

Le palais du roi de Butua [...] est gardé par des femmes noires, jaunes, mulâtres et blafardes... Elles étaient absolument nues, dénuées même du pagne qui couvre les parties de la pudeur chez les autres peuples de l'Afrique ; toutes étaient armées d'arcs et de flèches ; dès qu'elles nous virent, elles se rangèrent en haie, et nous laissèrent passer au milieu d'elles ${ }^{31}$.

Ces fortifications résument à elles seules l'état de barbarie et d'abrutissement dans lequel vivent les habitants. Elles établissent aussi les clivages qui sont au cœur de cette société et sur lesquels s'appuie le pouvoir : faible/fort, dominé/dominant, esclave/maître, femme/ homme. Ces femmes, barrières humaines protégeant l'accès de la maison du roi, n'ont d'autre valeur que le matériau que constitue leur corps. Et si ce sont elles qui ont été choisies pour cette fonction, c'est aussi parce que leur chair est réputée «plus filandreuse», plus dure donc, que celle des hommes (d'après les goûts cannibales). Elles n'en rempliront donc que mieux cette fonction de bouclier humain.

On peut mieux comprendre l'absence de considération dont sont victimes les femmes à Butua, lorsqu'on replace ce mépris dans la conception de la nature que développe Sarmiento et dont les mœurs des habitants sont l'incarnation vivante. Au cours d'un exposé théorique, Sarmiento avance l'idée de l'indifférence de la nature face à la vie. Toute sa longue description ethnographique des mœurs de Butua n'est

30 Sur l'usage sadien des savoirs ethnologiques des Lumières, voir Michel Delon, «Sade ethnologue» in À l'ombre des Lumières, littérature et pensée françaises au XVIII siècle, Ed. Trude Kolderup \& Svein-Eirik Fauskevåg, L’Harmattan, Paris, 2008, p. 203-212.

31 Sade, Aline et Valcour ou le roman philosophique, op. cit., p. 555. 
que la démonstration pratique de ce syllogisme : «La nature ne crée que pour corrompre : or, si elle ne se corrompt que par des vices, voilà le vice une de ses lois... voilà donc ce qui détruit l'homme, essentiel au plan général ${ }^{32} . »$ Pareillement, $l^{\prime}$ homme, étant une créature forgée par la nature, il naît mauvais et l'expression de cette méchanceté, réflexe accentué par la recherche du raffinement dans le mal, est la destruction, à commencer par celle de ses semblables. D'où ces expéditions guerrières gratuites, qui n'ont aucun but sinon le plaisir de saccager, auxquelles se livrent les chefs de Butua. Il est courant, explique Sarmiento : «de voir deux ou trois principaux chefs se réunir, pour aller, par seul principe d'amusement, saccager, détruire, incendier une bourgade, et en massacrer tous les habitants ${ }^{33}$.»

Cette pétition de principe qui fait de la guerre la principale industrie de l'homme naturel prolonge la pensée de Hobbes. Le mode de vie de ces sauvages, censé représenter la vraie nature de l'homme en l'absence de toute civilisation est la parfaite illustration des théories hobbesiennes sur les fins de l'homme, c'est-à-dire la poursuite d'un «agrément ${ }^{34} »$ ou plus fondamentalement, la poursuite de sa «propre conservation ${ }^{35} »$. Hobbes conclut, contrairement à Rousseau, à l'existence d'un droit de nature qu'il définit en ces termes : «la liberté qu'a chacun d'user comme il le veut de son pouvoir propre, pour la préservation de sa propre nature, autrement de sa propre vie, et en conséquence de faire tout ce qu'il considérera, selon son jugement et sa raison propres, comme le moyen le mieux adapté à cette fin $^{36}$.» Ainsi «chacun s'efforce de détruire ou de dominer l'autre ${ }^{37} »$. Hobbes termine le chapitre du Léviathan consacré à la «condition naturelle des hommes» par une conséquence éthique, fondamentale, de la "guerre de chacun contre chacun» : dans cet état «rien ne peut être injuste. Les notions de légitime et d'illégitime, de justice et d'injustice, n'ont pas ici

33 Ibid., p. 593.

34 Thomas Hobbes, Léviathan : traité de la matière, de la forme et du pouvoir de la république ecclésiastique et civile, Ed. François Tricaud, Paris, Sirey, 1983, p. 122.

35 Ibid., p. 122.

36 Ibid., p. 128.

37 Ibid., p. 122. 
leur place ${ }^{38} »$. Les seules «vertus» qui existent dans l'état de guerre sont «la violence et la ruse ${ }^{39} »$.

Ces idées de Hobbes concordent trop bien avec la réalité du système féodal pour qu'elles n'aient pas exercé un attrait sur Sade. De plus, l'amoralisme de ce système lui permet de jeter une nouvelle lumière sur ses goûts les plus inacceptables. Sade se fera donc une joie de mettre la condamnation de sa sexualité, qu'il vit sur le mode de rapports antagonistes et cruels, sur le compte de la bêtise de la société. Ce qui explique que, dans Aline et Valcour, texte qui apparemment ne ressemble en rien à la démesure d'une Nouvelle Justine, Sade ne peut s'empêcher de reprendre, dans le cadre du monde civilisé parisien, les motifs de domination et de lutte (à laquelle le vertueux succombe invariablement) dont Sainville avait été le témoin à Butua. Loin de l'Afrique des cannibales, la jeune et innocente Aline est la proie des ambitions de son père, le président de Blamont, qui a décidé qu'elle épouserait un de ses amis, financier, et, par ailleurs, son compagnon de débauche, le ridicule Dolbourg. Dans une lettre à ce dernier, le président de Blamont revient sur un épisode où Dolbourg s'est couvert de ridicule et il conçoit dorénavant la conquête du cœur de sa fille comme une entreprise militaire :

Il me paraît, mon cher Dolbourg, que jusqu'ici tes succès ne sont pas brillants... Si tu ne t'y prends pas mieux que cela, cependant, je crains que nous ne soyons réduits à emporter la citadelle d'assaut. Je t'aiderai à la battre en brèche, et pendant que tu formeras tes attaques, je te ménagerai des auxiliaires. Il arrive souvent que quand on a l'intention de se rendre maître d'une ville, on est obligé de s'emparer des hauteurs... ${ }^{40}$

À la lumière des voyages de Sainville, ces paroles doivent être prises au sens propre car l'état de guerre est une constante de l'humanité et aucune loi, aucune civilisation, aucune morale ne peut enrayer du cœur de l'homme le désir de détruire son semblable, fût-il son propre enfant. Et la cruauté, qui est l'expression de «la vie sauvage» ${ }^{41}$ est tout aussi présente dans la campagne enchanteresse de Vertfeuille que sous le soleil cuisant de Butua. En effet, Sade écrira à propos du Président : «Jamais je ne vis d'âmes si dures... Aussi n'est-ce pas pour rien qu'on

38 Ibid., p. 126.

39 Ibid., p. 126.

40 Sade, Aline et Valcour ou le roman philosophique, op. cit., p. 418.

41 Ibid., p. 645. 
[1']accuse d'avaler le gibier tout cru pour avoir le plaisir de le sentir palpiter sous [ses] dents ${ }^{42}$.» Le lecteur ne peut s'empêcher de voir dans cette phrase l'expression du sadisme même. Et ces mots portent bien la trace de la signature de Sade.

La volupté qui peut naître de la souffrance d'autrui est aussi clairement exprimée dans Isabelle de Bavière. Elle est associée à la guerre permanente dans laquelle la France avait sombré sous le règne de Charles VI. De même Jeanne Laisné et Adélaïde de Brunswick développent les conséquences néfastes des conflits armés pour des victimes innocentes du chaos politique. Entre Aline et Valcour et ces romans historiques, il n'y a apparemment que la distance du privé au public, de la nature à la culture, de l'individu à la politique. Or ces dichotomies perdent leur sens dans une vision où l'homme est un sujet engagé dans une guerre perpétuelle. Alors la «politique» que Sade envisage est la féodalité. Elle seule est la politique naturelle. En refusant l'hypocrisie du mensonge social sur la nature humaine, elle institue la force comme droit. Sade est avant tout un héritier de sa caste. Le système féodal sous lequel est né son nom, il l'assume non pas uniquement parce qu'elle lui permettrait de vivre ses goûts sans être inquiété, mais aussi parce qu'il croit sincèrement que l'homme est naturellement violent. Chez Sade, la philosophie du conflit, telle qu'elle prend forme dans ses œuvres signées, découle à la fois de son égoïsme, de sa lucidité et de son expérience personnelle. Ces textes constituent donc bien dans cette perspective une «otobiographie», au sens derridien. Ils sont aussi le testament, l'héritage que Sade lègue à ses fils — du moins à Louis-Marie, le militaire, son fils préféré dont il écrit qu'il «se distingue aux armées» — dans un geste symbolique pour faire perdurer l'honneur guerrier, associé au nom, dans un geste aussi d'éternel retour sur soi et vers ses origines.

ARMELLE ST-MARTIN

Université du Manitoba 